\title{
Erratum: Nonlocal Dynamics of Spontaneous Imbibition Fronts [Phys. Rev. Lett. 89, 104503 (2002)]
}

Dimitrios Geromichalos, Frieder Mugele, and Stephan Herminghaus (Received 11 September 2002; published 30 September 2002)

DOI: 10.1103/PhysRevLett.89.169904

PACS numbers: 47.55.Mh, 05.70.Ln, 61.30.Hn, 68.35.Ct, 99.10.+g

There is an error in the reproduction of Fig. 1(a). The scale labels should be micrometers $(\mu \mathrm{m})$, not meters $(\mathrm{m})$ as shown in the figure. 